\title{
Zwei Jahre Mitarbeit im Verein Junge Hausärztinnen und -ärzte Schweiz
}

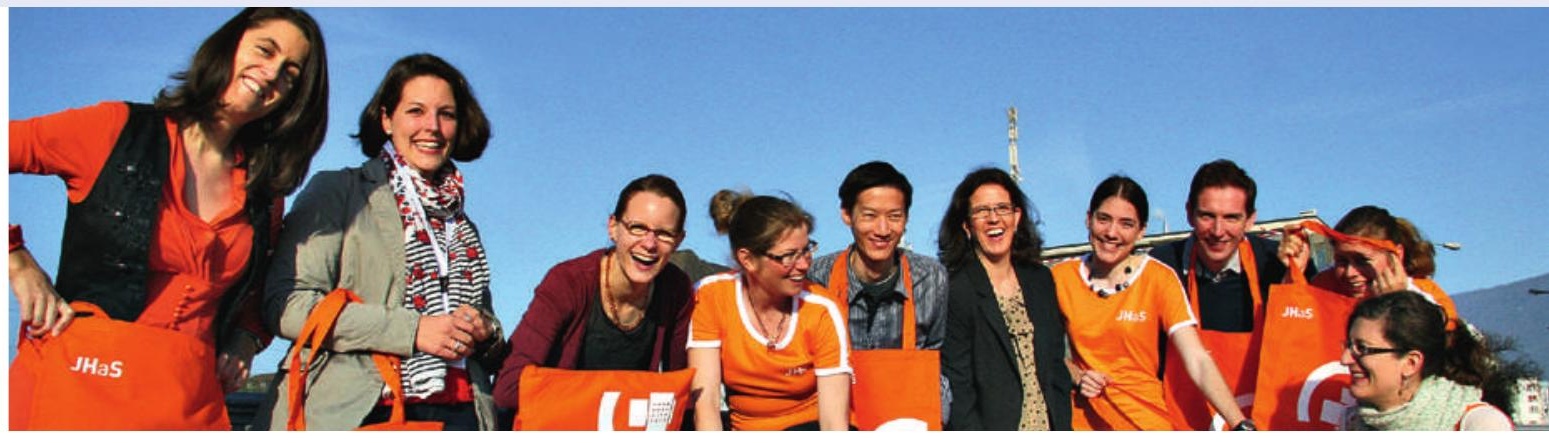

Sarah Singer

\footnotetext{
* scherzhaft für alle nicht JHaS-Mitglieder.
}

sarah.singer[at]jhas.ch
Commitment unlocks the doors of imagination, allows vision, and gives us the «right stuff» to turn our dreams into reality. (James Womack)

Es brauchte Zeit und Überwindung, sich in eine schon bestehende Gruppe einzubringen. So war es jedenfalls bei mir, als ich mit den Jungen Hausärztinnen und -ärzten Schweiz (JHaS) in Kontakt kam. Im Studium lernte ich sie über ihren Newsletter und den Berner Stammtisch kennen. Dabei hat mich dieser übermotivierte und etwas verrückte Haufen zugleich abgeschreckt und begeistert. Abgeschreckt, weil ich die Hälfte nicht verstand.

Da war die Rede von FMH, VSAO, MFE, SGAM, SGIM, SGP, GDK, JHaS, IHAMB, BIHAM, VBHM, VDGM, WONCA, MWS, DRG und WHM. Viele Namen wurden genannt, die ich noch nie gehört hatte, es wurde über DRG und Managed Care gesprochen, über einen neuen Facharzttitel (dabei wusste ich noch gar nichts über den alten) und über das Hippokrates-Programm.

Wenigstens schmeckte die Pizza am Stammtisch gleich wie überall! Aber begeistert war ich trotzdem. Da wurde Engagement und Freude für einen Beruf gezeigt, den ich zwar für mich immer mehr entdeckte, der aber sonst oft schlechtgemacht wurde und über den ich viel Negatives hörte. Dieser Kampfgeist, diese Motivation und Visionen haben mich angesteckt.

Ende April 2012 erhielt ich die erste E-Mail, Vorschläge, wie ich mich bei den JHaS beteiligen

Sarah Singer ist eine von 200 motivierten Mitgliedern der JHaS. Ihr Artikel beschreibt gut auch meine ersten Gefühle beim Treffen mit den engagierten künftigen Hausärzten: Fachbegriffe schrecken ab, die Diskussionen um BIP, Managed Care usw. interessieren zwar, aber man versteht zu wenig davon. Keine Sorge, das geht vorbei! Früher oder später setzen wir uns alle mit den Details des Grundversorgerseins auseinander. Da sind gute Bekannte und ein Netzwerk wichtig. Davon profitieren unsere Mitglieder bereits heute. Interessierte im Studium, in der Assistenzzeit und 5 Jahre nach Praxiseröffnung sind herzlich willkommen, auf www.jhas.ch Mitglied zu werden.

Dr. med. Sven Streit, Co-Präsident JHaS könnte. So viele Möglichkeiten! Auf ein kleines Engagement folgten mehrere. Auf die erste E-Mail folgten in den nächsten zwei Jahren weitere 1068 Mails - und manche Stunde Arbeit, die ich aber selten als solche wahrnahm. Und was hat mir das gebracht?

\section{Austausch und Möglichkeiten}

Am wertvollsten sind die Kontakte - am Stammtisch, an Kongressen, via E-Mails und in persönlichen Gesprächen, mit Jungen wie mit Älteren, in der Schweiz und europaweit. Ich hatte die Möglichkeit ein Netzwerk aufzubauen, in dem ich bei fast jedem Anliegen einen Ansprechpartner finde. Ich lerne nicht nur andere Meinungen kennen, ich kann auch die Meinung von uns Jungen ausdrücken, z. B. an Sitzungen, Kundgebungen oder bei einer FotoAktion für die Presse. Und mit dem Wunsch, Hausarzt zu werden, steht man nicht mehr alleine da, sondern bespricht zusammen die Freuden und manchmal auch den Ärger. In diesen zwei Jahren hatte ich zahlreiche Möglichkeiten, die ich ohne die JHaS nicht gehabt hätte. So konnte ich unter anderem das Gastrecht bei einigen Vorstandssitzungen des MFE (Médecins de Famille et de l'Enfance) nutzen, einen Artikel für die SÄZ schreiben, helfen, einen JHaS-Kongress auf die Beine zu stellen, und an diesem Kongress einen Talk moderieren.

\section{Spass und noch ein bisschen mehr}

An den Sitzungen kann man Spass haben, beim Fotomachen sowieso. Kongresspartys werden spätestens dann, wenn die «AHaS»* das Weite gesucht haben, richtig lustig, und ein JHaS-Ausflug ist wunderbar.

Als JHaS-Mitglied kann man auch den einen oder anderen Vorteil geniessen, wenn es um Stellensuche oder um Forschungsprojekte geht. Und heute kenne ich den Wortsalat sogar auf Französisch: FMH, ASMAC, MFE, SSMG, SSMI, SSP, CDS, JHaS, IUMG, IMC, VDGM, WONCA, MWS, DRG und FMF ... 